\title{
Numerical model of electrode induction melting for gas atomization
}

\author{
Valdis Bojarevics, Alan Roy and Koulis Pericleous \\ Centre for Numerical Modelling and Process Analysis, \\ CMS, University of Greenwich, London, UK
}

\begin{abstract}
Purpose - The purpose of this paper is to create a numerical model of electrode induction melting process for the gas atomization (EIGA) and process and investigate the complex interaction of the electromagnetic and thermal fields on the fluid flow with free surface.

Design/methodology/approach - The modelling approach is based on the free surface code SPHINX which includes time dependent electromagnetic, thermal and fluid flow with free surface modelling and the commercial software COMSOL for investigating 3D electromagnetic effects.

Findings - The melting dynamics, liquid film formation and the outflow free surface behavior are predicted by SPHINX using an optimized geometry. Quasi-stationary AC electromagnetic solutions with COMSOL predict some 3D effects of the coil, including frequency dependent estimates of voltage, electric current and power.

Originality/value - The importance of magnetic forces controlling the free surface jet formation, partial semi-levitation and the outflow superheat is uncovered by numerical modelling tools. An optimized geometry is presented for the EIGA process.
\end{abstract}

Keywords Induction melting, Magnetohydrodynamics, Free surface dynamics, AC electrodynamics, Titanium powder production

Paper type Research paper

\section{Introduction}

There is an increased demand for high-quality metallic powders used in production of advanced materials for aerospace, automotive, biomedical and other applications (Antony and Reddy, 2003; Belyanchikov, 2008; Gerling et al., 2002; Dinda et al., 2008). Production of reactive high melting temperature metal powders requires a contactless technique avoiding direct contact to refractory crucible walls. Direct melting of an electrode by induction heating is a contactless technique suitable for reactive high melting temperature alloys ( $\mathrm{Ti}, \mathrm{Nb}, \mathrm{Zr}$, etc.). The technique has found application in production of titanium alloy powders by gas atomization, known as electrode induction melt inert gas atomization (EIGA) (Gerling et al., 2002; Franz et al., 2008). An alternative method can be used to produce micro-droplets directly, without gas atomization, by an electromagnetic droplet generator device described in Shimasaki et al. (2009).

Electrodes up to $10-15 \mathrm{~cm}$ in diameter can be melted in a semi-continuous EIGA process using prefabricated cylindrical ingots - typically obtained at relatively low cost using vacuum arc remelting. The electrode bottom is immersed in an induction coil where it melts and produces dripping or streaming liquid metal. The dynamics of the melting process are complex, involving continuous adjustment of the electromagnetic field due to propagation of the melt front and motion relative to the coil.

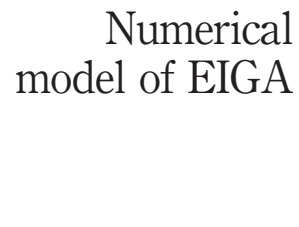

1455 
COMPEL 30,5

1456
The melt flow is controlled by the melting rate, gravity force in the liquid film and the electromagnetic forces. The high frequency coil current $(100-200 \mathrm{kHz})$ creates the induced current layer in the electrode, which is spatially non-uniform along the surface. The liquid film is confined by an electromagnetic "pinching" force, but the non-uniformity of this force along the surface creates a rather strong electromagnetically driven flow. This type of flow can, in some cases, oppose the dripping of the film flow due to gravity, and lead to an interruption of the melt stream. A quasi-steady state is desirable, avoiding unstable dripping and maintaining a conically symmetrical shape at the electrode bottom. In order to stabilize the circumferential uniformity, the ingot is rotated at low angular velocities of 5-12 turns per minute. This means that angular momentum transport in the conical melt film flow must be included in the model.

\section{Modelling description}

The numerical simulation technique previously used for magnetic levitation and cold crucible modelling (Bojarevics et al., 2004, 2000) is applied to the study of electrode induction melting. The numerical code SPHINX has been modified to permit time dependent modelling of the electromagnetic, thermal and fluid flows coupled with the changing free surface.

We consider an axisymmetric approximation of the ingot and molten film flow with the detailed model representation given in previous publications (Bojarevics et al., 2004, 2000). The boundary conditions at the external free surface of the viscous turbulent fluid flow are stated for the stress tensor $\Pi$ on the surface:

$$
\Pi_{n n}=\gamma K, \quad \Pi_{n \tau}=\gamma_{T} \nabla T \cdot \mathbf{e}_{\tau},
$$

where $\gamma(T)=\gamma\left(T_{m}\right)+\gamma_{T} \cdot\left(T-T_{m}\right)$ is the temperature dependent surface tension coefficient above the melting temperature $T_{m} ; K$ - the local mean curvature of the surface, and the subscripts $n, \tau$ correspond to projections onto $\mathbf{e}_{\mathrm{n}}$ and $\mathbf{e}_{\tau}-$ the normal and tangent unit vectors at the free surface. In addition to the dynamic conditions (1), the time dependent fluid velocity $\mathbf{v}(\mathrm{t})$ continuously adjusts the interface, given mathematically as the local radius vector on the surface $\mathbf{R}(\mathrm{t})$ :

$$
\mathbf{e}_{\mathbf{n}} \cdot \mathbf{v}=\mathbf{e}_{\mathbf{n}} \cdot \partial_{\mathrm{t}} \boldsymbol{R}
$$

The thermal boundary conditions at the melt free surface and on the ingot surface are the radiation loss:

$$
\boldsymbol{- n} \cdot(-k \nabla T)=\varepsilon \sigma\left(T_{a m b}^{4}-T^{4}\right) .
$$

The dynamic interactions of the turbulent flow with the moving interface, confined by the surface tension and the magnetic force, are analysed using a unified numerical model for the time dependent liquid metal and magnetic field generating coil system. The governing equations are solved numerically by use of the SPHINX code based on continuous transformation functions for the shape change in the physical space. The electromagnetic force is computed in the SPHINX model using the integral equation representation for the axisymmetric shape of the metal and coil. The modified $k$ - $\omega$ turbulence model, including magnetic damping terms, is used to describe the mixing and damping properties at smaller scales not resolved by the macro model. A spectral-collocation method is used to solve the resulting equations, according to 
which the velocity vector components and pressure are represented as series of Chebyshev polynomials and Legendre functions.

The commercially available software COMSOL is used to check the validity of the solutions in quasi-stationary states, to predict some 3D effects of the coil and the relationship between voltage and current.

\section{Comsol model results}

3D and 2D axisymmetric solutions with fixed geometries showing the electromagnetic field near the electrode and liquid metal film were produced using the finite element software COMSOL. The results were in good agreement with those produced independently by SPHINX.

The 3D solution in Figure 1 shows surface current density and magnetic streamlines flowing near a conically shaped titanium ingot immersed in a tapered three-turn coil (complete with supply leads).

The current flows in a thin skin and concentrates on the inner surfaces of the coil due to confinement of magnetic flux. It is useful to compare this solution with the equivalent axisymmetric representation in Figure 2 which shows $|\mathrm{B}|$ in the air. The position of the coil and the actual shape of the melted ingot (which is slightly different from Figure 1) corresponds to geometry obtained from the transient SPHINX simulation at a particular time step (Figures 5, 8 and 10). The averaged axisymmetric approach is sufficiently accurate to represent the effect of the full 3D electromagnetic field in the titanium, which is the main focus for this modelling.

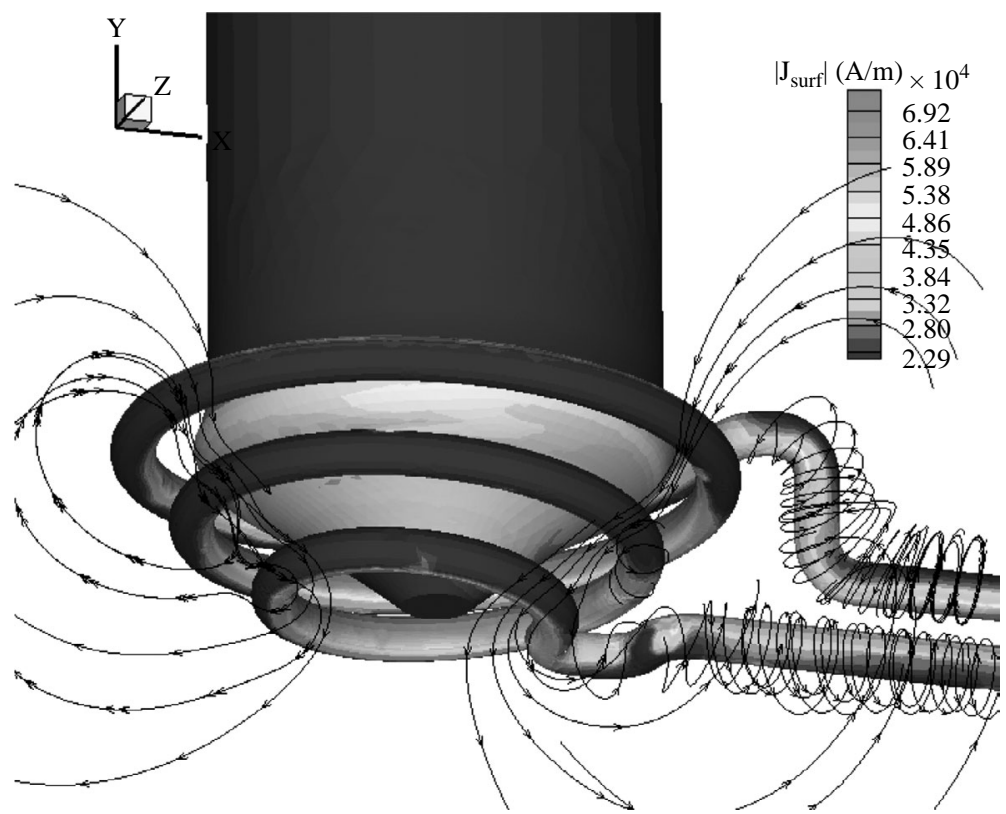

Notes: Image computed with COMSOL; effective voltage $525 \mathrm{~V}, \mathrm{f}=200 \mathrm{kHz}$

\section{Numerical model of EIGA}

1457
Figure 1.

3D solution for the coil and conically shaped ingot showing streamlines of magnetic flux and scalar plot of surface current density $|J|$ 


\section{COMPEL 30,5}

\section{8}

Figure 2.

2D axisymmetric solution showing scalar map of magnetic field $|\mathrm{B}|$ in the air and streamlines of magnetic flux for the coil current $\mathrm{I}_{\mathrm{eff}}=1.4 \mathrm{kA}$ at $\mathrm{f}=200 \mathrm{kHz}$

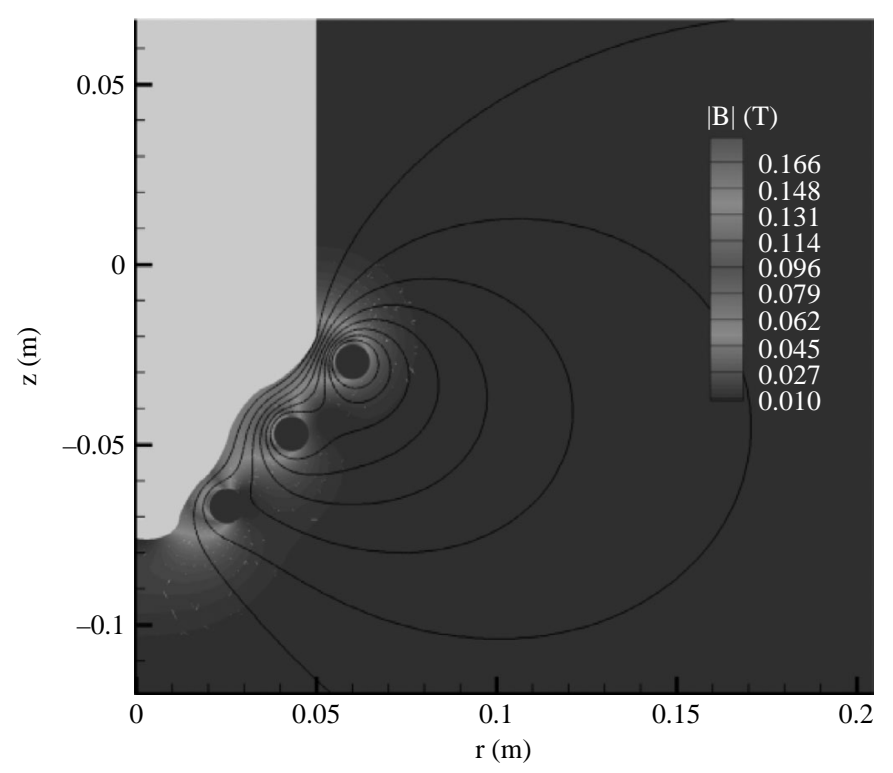

Note: Image computed with COMSOL

COMSOL can be used to deduce the complex voltage phasors needed to drive the required effective current in the coil for the particular geometrical arrangements in Figures 1 and 2. In the axisymmetric solution, the loop voltage for each turn is represented by a complex phasor. To drive an effective current $\mathrm{I}_{\mathrm{eff}}=1.4 \mathrm{kA}$ at $\mathrm{f}=200 \mathrm{kHz}$, the respective voltage phasors were $(19.305+357.958 \mathrm{i}, 14.0392+269.828 \mathrm{i}, 8.972+114.2096 \mathrm{i})$ (in volts). This gives the net voltage $\mathrm{V}=42.317+741.996 \mathrm{i}(\mathrm{V})$ or effective voltage of $\mathrm{V}_{\text {eff }}=525 \mathrm{~V}$. The computed Joule loss in the titanium ingot $\mathrm{Q}_{\mathrm{Ti}}=33.8 \mathrm{~kW}$ while the Joule loss in the coils starting with the top, was $3.6,2.7$ and $1.9 \mathrm{~kW}$, respectively. This implies a power efficiency of 0.8 . The computed power loss in the titanium matches the value obtained by the SPHINX solution $\mathrm{Q}_{\mathrm{Ti}}=33.6 \mathrm{~kW}$ (Figure 10).

Figures 3 and 4 were produced using the fixed axisymmetric geometry in Figure 2. When an effective current of $1.4 \mathrm{kA}$ is maintained in the coils, the graphs indicate that the total power supplied to the load and the coil grows as square root of the frequency, and that the effective voltage grows linearly with the frequency. An interesting conclusion follows from Figure 4, which is that the electrical efficiency reaches a saturation of about 0.8 above $50 \mathrm{kHz}$.

Attempts at using COMSOL to predict the thermal and velocity fields were hampered by the presence of intense turbulent flow in the melt zone and these difficulties were not completely resolved with the current version of the software.

\section{Sphinx results for the melt dynamics}

The axisymmetric approximation is a reasonable choice for the liquid metal region. The integral equation formulation used in the SPHINX code avoids the need to mesh the surrounding air volume; instead the mesh used for the fluid dynamics can be 


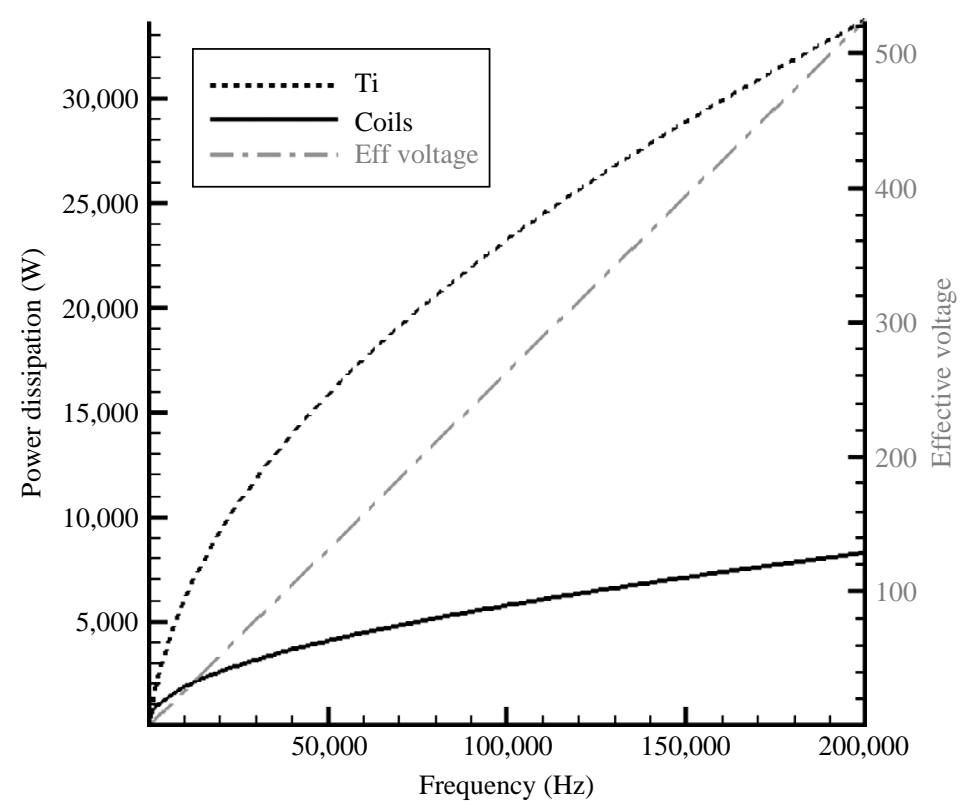

\section{Numerical model of EIGA}

1459

Figure 3.

Time-averaged power dissipation in the titanium load, in the coil and the effective voltage as a function of frequency when the coil effective current of $1,400 \mathrm{~A}$ is maintained

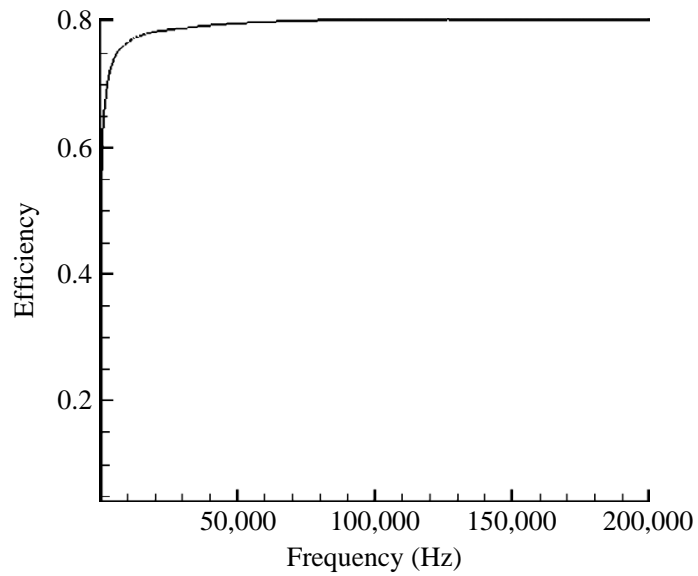

Figure 4. Efficiency when $\mathrm{I}_{\text {eff }}=1,400 \mathrm{~A}$

directly used for the electromagnetic field calculations in the fluid. The additional current carrying regions in the coil need to be approximated as axisymmetric. The validity of this approach is supported by the high level of agreement between estimates of $|\mathrm{J}|$ and power dissipation in the titanium produced by COMSOL compared to those produced by SPHINX (at a particular time step). The SPHINX model follows the dynamic evolution of the liquid surface supported by the magnetic force and the associated velocity field. The computed position of the liquid metal surface is determined by the complex interaction of the induced electromagnetic field in various regions and the effects of fluid flow. This is a complex dynamic process where various 
COMPEL 30,5

1460 properties are important, not least the internal fluid flow turbulence with the magnetic interaction. The flow intensity is strongly dependent on the electromagnetic field penetration depth which varies according to the frequency of the AC supply. The cases considered in this section were run at suitably high frequencies of $150 \mathrm{kHz}, 200 \mathrm{k} \mathrm{Hz}$ to meet the power requirements for a melt rate $\Gamma=120 \mathrm{~kg} / \mathrm{h}=0.033 \mathrm{~kg} / \mathrm{s}$. A higher frequency $\mathrm{AC}$ field is preferable because of the lower penetration to the liquid domain, concentrating the magnetic confinement force near the surface and stabilizing it. Figure 5 shows a full view of the titanium ingot used in the numerical model and a typical solution. The instantaneous fluid flow and temperature distribution in the thin molten layer is driven by gravity and the electromagnetic force distribution created by the induced $\mathrm{AC}$ current from the coil carrying $\mathrm{I}_{\mathrm{eff}}=1,400 \mathrm{~A}$ at $200 \mathrm{kHz}$.

The solution is time dependent accounting for the gradual melting of the titanium ingot and the upward motion of the coil along the solid part of the ingot. The propagation speed of the coil is computed according to the prescribed melting rate:

$$
w_{c}=\frac{\Gamma}{\rho \pi r_{c}^{2}}=\frac{0.033}{4,110 \cdot \pi \cdot 0.05^{2}}=0.001\left(\frac{\mathrm{m}}{\mathrm{s}}\right)
$$

The ingot, which is initially a solid cylinder, is heated inductively until a thin layer of material at the bottom of the hemispherical surface reaches the melting temperature. When this condition is met, the hydrodynamic part of the model is activated. Figures 6 and 7 show the hydrodynamic and electrodynamic fields at an early stage in development after only $25 \mathrm{~ms}$. At this stage, the flow rushes down to fill the space enclosed by the lowest coil, forming a shape which is not yet conical. The dashed lines in Figure 7 show the initial position of the coil when the ingot was still solid. The total
Figure 5.

View of the titanium ingot and the instantaneous fluid flow and temperature in the molten layer for the three-turn coil carrying $\mathrm{I}_{\mathrm{eff}}=1,400 \mathrm{~A}$ at $200 \mathrm{kHz}$

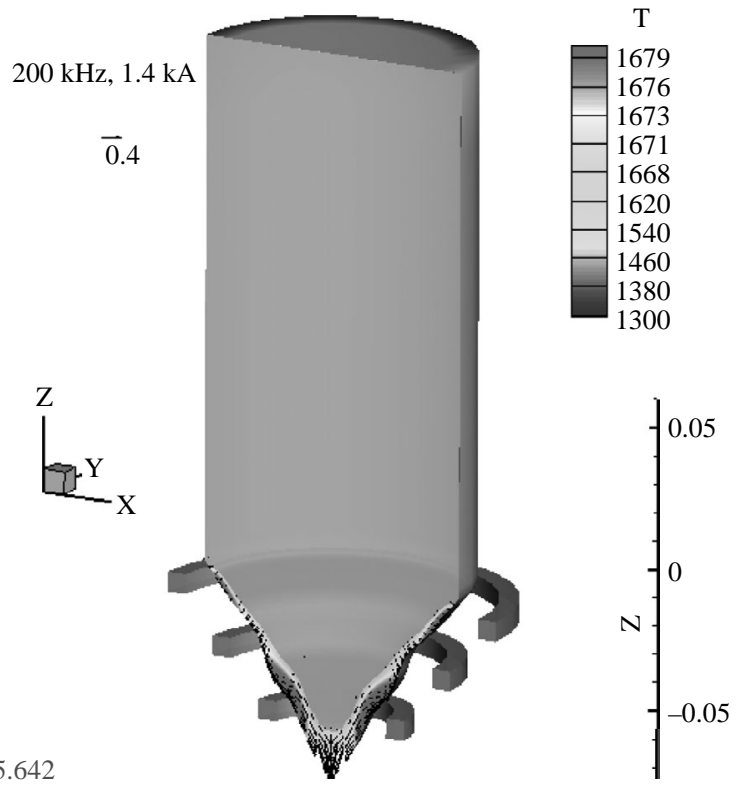




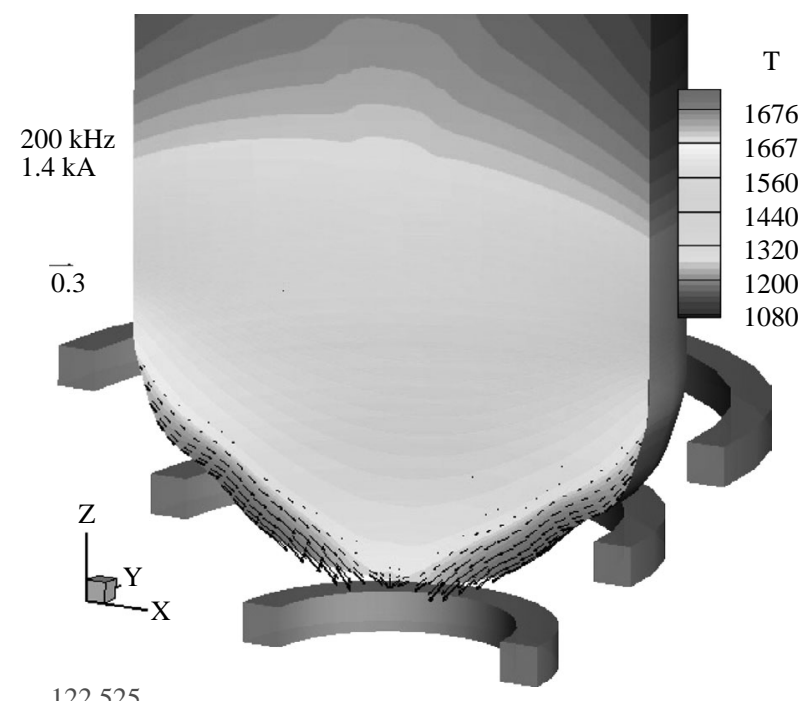

Electric current and force

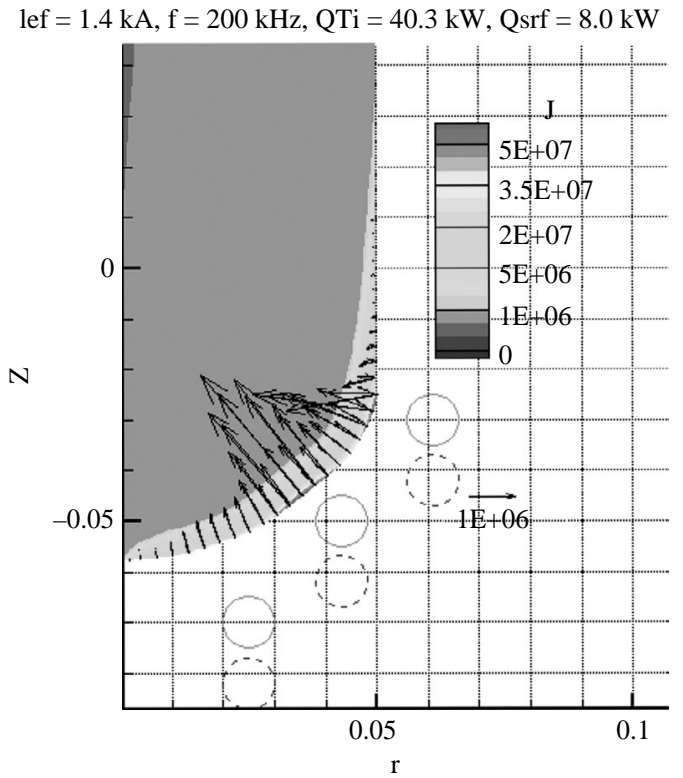

Figure 6.

Instantaneous fluid temperature and flow driven by gravity and the electromagnetic force distribution in the titanium load at initial time when melting starts for the coil $\mathrm{I}_{\text {eff }}=1,400 \mathrm{~A}$

Figure 7.

Time-averaged electric current and electromagnetic force distribution in the titanium load at initial time when melting starts for the coil $\mathrm{I}_{\text {eff }}=1,400 \mathrm{~A}$, $\mathrm{f}=200 \mathrm{kHz}$

Joule heating for the titanium material $\mathrm{Q}_{\mathrm{Ti}}$ and the radiation loss over the total ingot surface $Q_{\text {srf }}$ are indicated for this particular moment in time. (Figures 6 and 7 and the following ones are zoom-in views of the total solution domain, as in Figure 5, in order to show the details of the thin liquid region.) 
COMPEL 30,5

1462
After a few seconds evolution of the fully coupled electromagnetic, thermal and flow fields, a quasi-steady conically shaped solid core is formed, along which molten liquid metal flows. The flow is mainly downwards due to the prevalent action of gravity (Figure 8). Some dynamically waving features on the surface are observed, partially corresponding in places to the localized action of the coil current carrying elements. The flow computation proceeds in adjustable time steps from 0.125 to $1 \mathrm{~ms}$. The results in Figure $8\left(\mathrm{I}_{\text {eff }}=1,400 \mathrm{~A}\right)$ are compared with those obtained using a slightly higher coil current $\left(\mathrm{I}_{\text {eff }}=1,600 \mathrm{~A}\right)$, shown in Figure 9. Increasing the current leads to a stronger magnetic confinement force and results in a narrower exit jet. Another important feature is that it produces an upwards-directed vortex flow near the surface (Figure 9). The vortex is generated by the differential action of the electromagnetic force in the skin layer. The presence of the vortex increases the local liquid film thickness in this region and leads to a rise in localized heating due to longer residence time of fluid in the region of maximum Joule heating. The final outflow jet is still rather stable, but its size is more constrained by the electromagnetic "pinch" effect.

The electromagnetic field distribution for the previous two cases is compared in Figures 10 and 11. As expected, the Joule heating increases with the higher current of $1.6 \mathrm{kA}$, but the total radiation loss stays approximately the same at the final quasi-stationary stage. This means that a higher superheat of the liquid metal outflow can be obtained with careful adjustment of the coil current and frequency. The examples shown in Figures 8-11 represent optimized cases and were only discovered after performing a large number of less successful numerical experiments. An example of an "unsuccessful" test run is seen in Figure 12, where the bottom of the electrode is molten flat and the last remaining liquid is dripping off in the middle. Another example of an unacceptable melting regime is shown in Figure 13 using the reduced frequency of $150 \mathrm{kHz}$. In the last example the superheat is rather low, and the outflow jet temperature is just above the melting temperature.
Figure 8.

A quasi-stationary temperature and fluid flow distribution in the molten titanium electrode after few seconds of the coupled development for the coil $\mathrm{I}_{\text {eff }}=1,400 \mathrm{~A}, 200 \mathrm{kHz}$

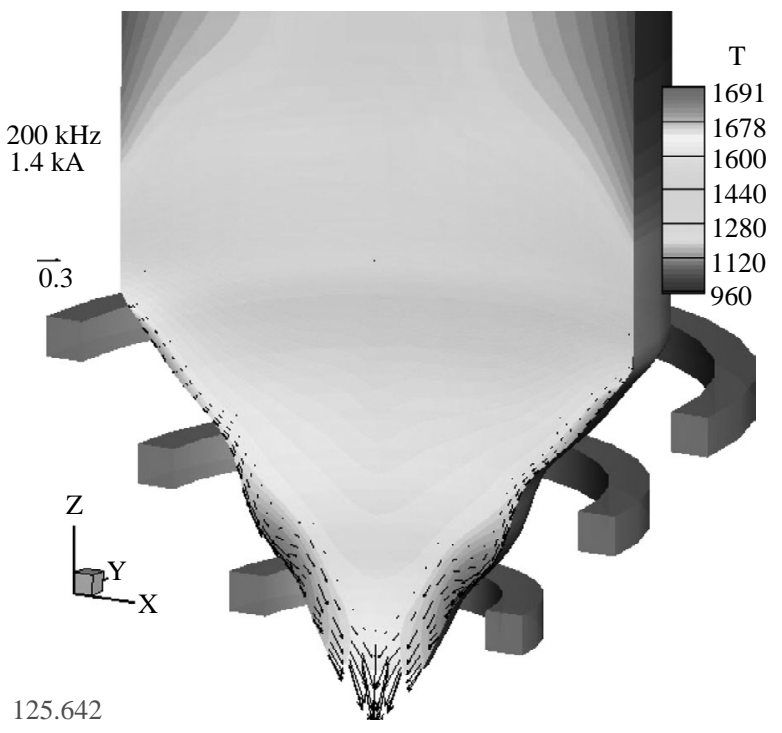



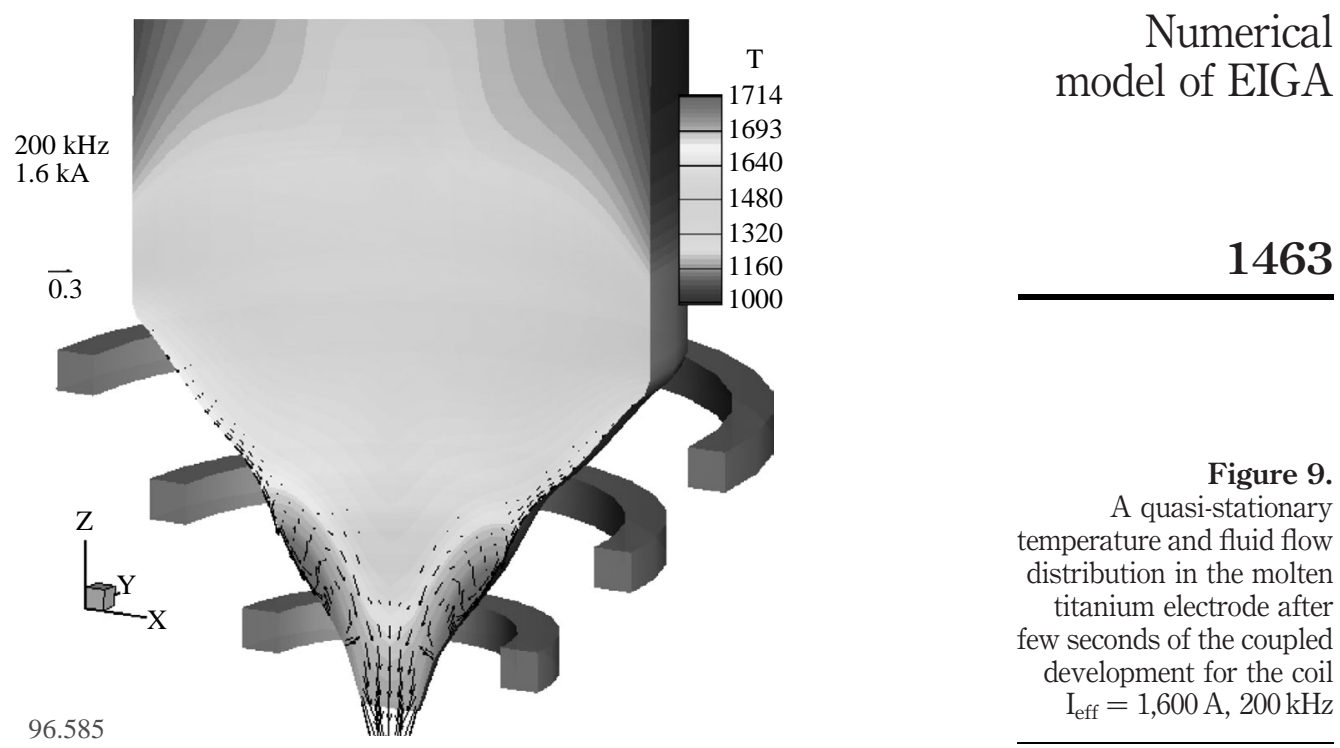

1463

Electric current and force

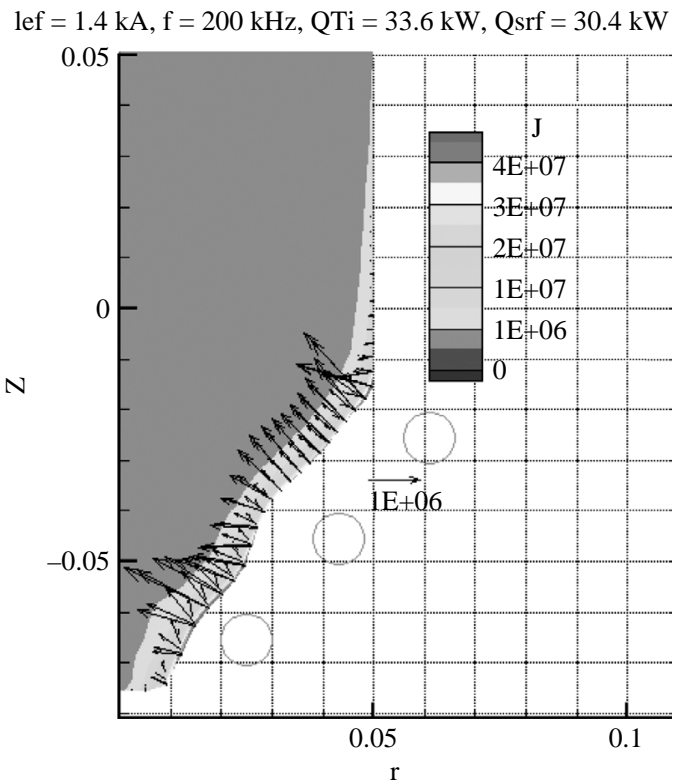

Figure 9.

A quasi-stationary temperature and fluid flow distribution in the molten titanium electrode after few seconds of the coupled development for the coil $\mathrm{I}_{\text {eff }}=1,600 \mathrm{~A}, 200 \mathrm{kHz}$

Figure 10.

The electric current and electromagnetic force distribution in the titanium load after few seconds of the coupled development for the coil $\mathrm{I}_{\text {eff }}=1,400 \mathrm{~A}, 200 \mathrm{kHz}$

\section{Conclusions}

Numerical models for the melting dynamics in the EIGA process have been developed and applied to predict an optimized melt outflow, its stability and temperature. It is demonstrated that the electromagnetically created internal flow features can partially 


\section{COMPEL 30,5}

\section{4}

\section{Figure 11.}

The electric current and electromagnetic force distribution in the titanium load after few seconds of the coupled development for the coil $\mathrm{I}_{\mathrm{eff}}=1,600 \mathrm{~A}, 200 \mathrm{kHz}$

\section{Figure 12.}

An "unsuccessful" melting example at the last stage before solution collapse
Electric current and force

lef $=1.6 \mathrm{kA}, \mathrm{f}=200 \mathrm{kHz}, \mathrm{QTi}=36.8 \mathrm{~kW}$, Qsrf $=30.5 \mathrm{~kW}$

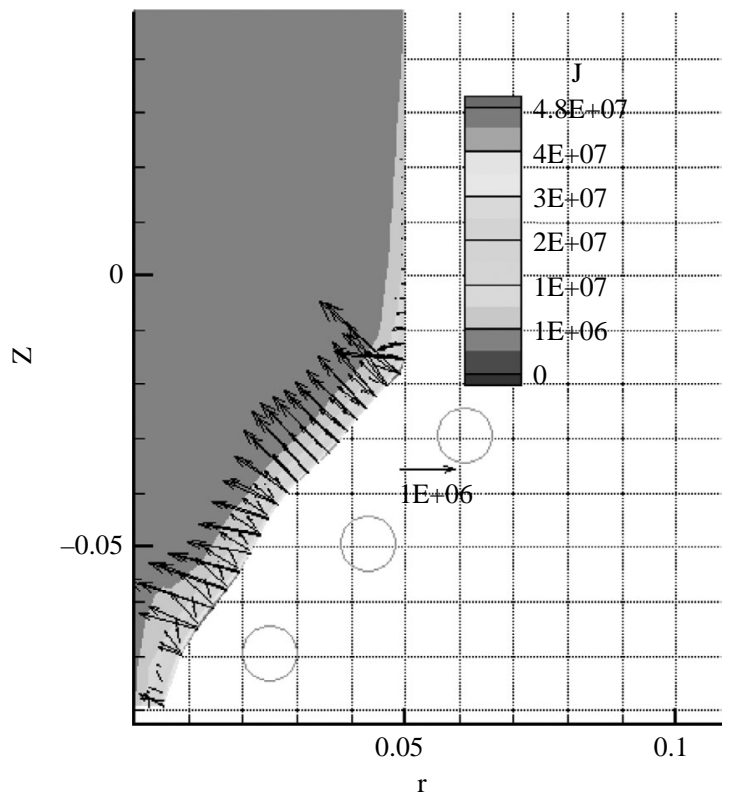

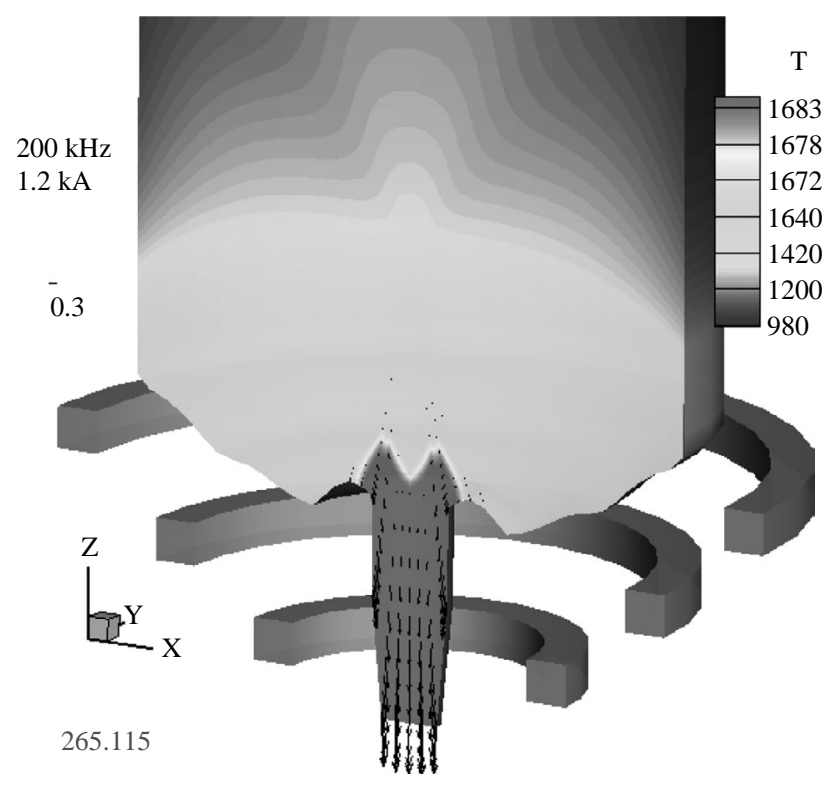




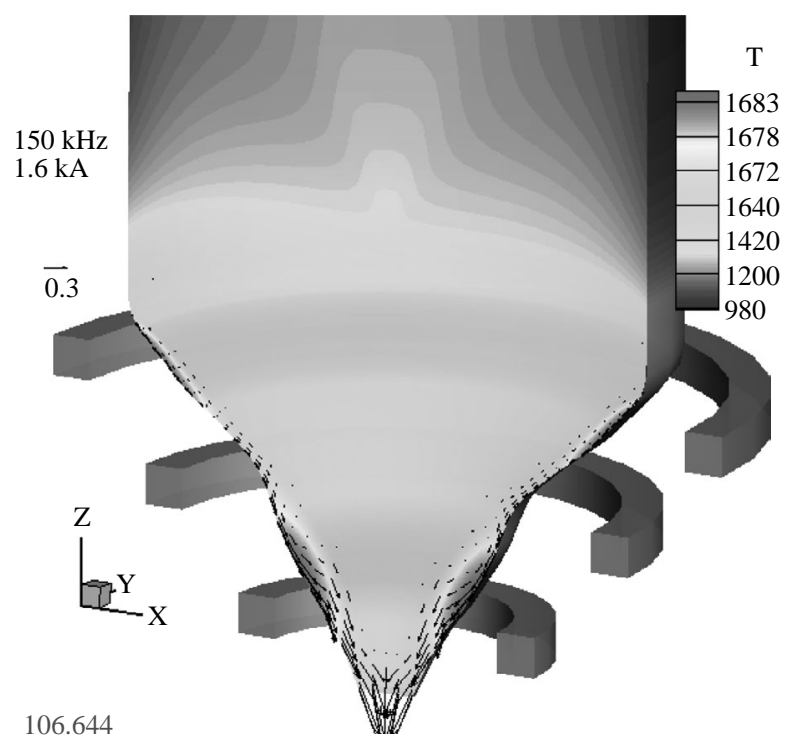

semi-levitate the melt film, increase the liquid residence time in the high heating zone and constrain the outflow in a narrow well-defined jet.

\section{References}

Antony, L.V.M. and Reddy, R.G. (2003), "Processes for production of high-purity metal powders", J. of Metals, Vol. 55 No. 3, pp. 14-18.

Belyanchikov, L.N. (2008), "Special electrometallurgy of promising structural materials based on titanium aluminides", Russian Metallurgy (metally), Vol. 2008 No. 3, pp. 9-22.

Bojarevics, V., Pericleous, K. and Cross, M. (2000), "Modelling the dynamics of magnetic semi-levitation melting”, Metallurgical and Materials Transactions B, Vol. 31, pp. 179-89.

Bojarevics, V., Harding, R.A., Pericleous, K. and Wickins, M. (2004), "The development and validation of a numerical model of an induction skull melting furnace", Metallurgical and Materials Transactions B, Vol. 35, pp. 785-803.

Dinda, G.P., Song, L. and Mazumder, J. (2008), "Fabrication of Ti-6Al-4V scaffolds by direct metal deposition”, Metallurgical and Materials Transactions A, Vol. 39, pp. 2914-22.

Franz, H., Plochl, L. and Schimansky, F.-P. (2008), "Recent advances of titanium alloy powder production by ceramic-free inert gas atomization", Proc. Titanium 2008, International Titanium Association, Las Vegas, NV, USA.

Gerling, R., Schimansky, F.P., Wegmann, G. and Zhang, J.X. (2002), "Spray forming of Ti48.9Al and subsequent hot isostatic pressing and forging", Materials Sci. Eng., Vol. A326, pp. 73-8.

Shimasaki, S., Imanishi, K., Taniguchi, S. and Bojarevics, V. (2009), "Manufacturing of uniformly sized silicon particles for solar cell from molten metal jet by electromagnetic pinch force", Proc. 6th Int. Symp. Electromagn. Processing Materials, ISIJ, Dresden, Germany, pp. 899-902. 
COMPEL

30,5

1466

\section{About the authors}

Valdis Bojarevics studied at the University of Latvia, obtained $\mathrm{PhD}$ at St Petersburg Technical University in 1982 and DrSci (Dynamics of Fluids) at the Latvian Academy of Sciences in 1992. He joined the University of Greenwich in 1995 and became the Reader of Magnetohydrodynamics in 2005. He has published over 100 scientific papers and co-authored three books. Valdis Bojarevics is the corresponding author and can be contacted at: v.bojarevics@gre.ac.uk

Alan Roy is a Research Fellow in the Centre for Mathematical Modelling and Process Analysis at the University of Greenwich. He has a background in Applied Mathematics and Computational Electromagnetics.

Koulis Pericleous studied Aeronautical Engineering at Queen Mary College, London University where he obtained a $\mathrm{BSc}$ and then a $\mathrm{PhD}$ on the subject of Unsteady Aerodynamics in 1977. He joined the University of Greenwich in 1989 and became the Professor of CFD in 1994. He has published over 200 scientific papers and supervised successfully $24 \mathrm{PhD}$ students. 